\title{
Quality of life and stress level in adolescents with idiopathic scoliosis subjected to conservative treatment
}

\author{
E Kinel $^{{ }^{*}}$, T Kotwicki ${ }^{2}$, A Podolska ${ }^{1}$, M Białek $^{3}$, W Stryła $^{1}$ \\ From 9th International Conference on Conservative Management of Spinal Deformities - SOSORT 2012 \\ Annual Meeting \\ Milan, Italy. 10-12 May 2012
}

\section{Background}

The quality of life and stress level of adolescents suffering from idiopathic scoliosis and submitted to brace treatment is the object of increasing interest of the professionals.

\section{Aim}

The aim of the study was to evaluate the quality of life and stress level in adolescents with idiopathic scoliosis who are under brace treatment.

\section{Methods}

It involved 45 adolescents, ages ranging between 10.0 and 15.0 years, all with Adolescent Idiopathic Scoliosis (AIS), with Cobb angle between 20-45 degrees. The adolescents were wearing the same kind of brace (Chêneau orthosis) for more than 3 months for at least $12 \mathrm{~h}$ per day. Two questionnaires were used: the Brace Questionnaire (BrQ) and Bad Sobernheim Stress Questionnaire (BSSQ). The $\mathrm{BrQ}$ is an instrument for measuring quality of life of scoliotic adolescents who are being treated conservatively with wearing of a corrective brace. The BrQ consists of 34 Likert-scale items associated with eight domains. The BSSQ (Brace and Deformity) questionnaire estimates the stress scoliosis patients have whilst wearing their brace. The analysis considered the type of treatment, curve location, correlation of the total score with age, Cobb angle and Bunnell rotation angle.

\section{Results}

The age was $13.6 \pm 1.3$ years. Cobb angle was $31.7 \pm 7.6$ degrees. The mean score for BrQ was $78.1 \pm 11.3$ points. Adolescents revealed higher score with BSSQ Deformity (median $=15$ ) comparing to BSSQ Brace $($ median $=12)$.

\section{Conclusion}

Conservative treatment does not severely impact on the quality of life of scoliotic adolescents. The adolescents who were under brace treatment suffered moderate level of stress from the deformity.

\section{Author details}

${ }^{1}$ Department of Rehabilitation, University of Medical Sciences, Poznan, Poland. ${ }^{2}$ Department of Paediatric Orthopaedics and Traumatology, University of Medical Sciences, Poznan, Poland. ${ }^{3}$ FITS Center, Jawor, Poland.

Published: 3 June 2013

\section{References}

1. Vasiliadis E, Grivas TB, Gkoltsiou K: Development and preliminary validation of Brace Questionnaire (BrQ): a new instrument for measuring quality of life of brace treated scoliotics. Scoliosis 2006, 1:7.

2. Botens-Helmus C, Klein R, Stephan C: The reliability of the Bad Sobernheim Stress Questionnaire (BSSQbrace) in adolescents with scoliosis during brace treatment. Scoliosis 2006, 1:22.

doi:10.1186/1748-7161-8-S1-061

Cite this article as: Kinel et al:: Quality of life and stress level in adolescents with idiopathic scoliosis subjected to conservative treatment. Scoliosis 2013 8(Suppl 1):O61. 